Ronald R. Swaisgood • Matthew P. Rowe •

Donald H. Owings

\title{
Antipredator responses of California ground squirrels to rattlesnakes and rattling sounds: the roles of sex, reproductive parity, and offspring age in assessment and decision-making rules
}

Published online: 18 December 2003

(C) Springer-Verlag 2003

\section{Behav Ecol Sociobiol (2003) 55:22-31}

Owing to an unfortunate oversight, the published version of the Results and Discussion sections of this paper did not take into consideration the journal's requirements with regard to the reporting of the outcome of statistical tests. After copy-editing, these sections should read as follows (Pages 26, 27, 28):

The online version of the original article can be found at http://dx.doi.org/10.1007/s00265-003-0684-2

R. R. Swaisgood (®)

Animal Behavior Graduate Group,

University of California,

Davis, CA 95616, USA

e-mail: rswaisgood@sandiegozoo.org

Tel.: +1-619-7443372

Fax: +1-619-7443346

M. P. Rowe

Department of Biology,

Appalachian State University,

Boone, NC 28608, USA

D. H. Owings

Department of Psychology and Animal Behavior Graduate Group,

University of California,

Davis, CA 95616, USA

Present address:

R. R. Swaisgood, Center for Reproduction of Endangered Species, Zoological Society of San Diego,

P.O. Box 120551, San Diego, CA 92112, USA 
is clearly related to potential risk (cf. Curio and Regelmann 1985). For the same reason, we also used average distance to the playback speaker as a measure of risk-taking in the playback study. This measure perhaps reflects even greater risk because the squirrel is entering the domain of a highly dangerous snake whose location has not yet been verified visually. Finally, we also examined the duration of the reapproach to the speaker because squirrels that rush back in quickly are at greater risk of coming within strike range before determining the location of the snake whose rattle it just heard. We should point out that although in reality no snake is present, squirrels displaying bolder behavior are in fact being more assertive, and would be much more likely to engage the snake had one been present.

The methods for the playback study are described above. For details of the tethered snake study we refer the reader to our earlier paper (Swaisgood et al. 1999a), but provide a brief summary here. The site, trapping and marking regime, identification of reproductive condition, and so forth are the same as for the playback study. We used four large rattlesnakes $(562-1150 \mathrm{~g})$ in the tethering trials, presenting one to each of the eight maternal squirrels. Approximately $1 \mathrm{~m}$ from the subject's natal burrow we drove a stake into the ground and attached the snake to a $40-\mathrm{cm}$ monofilament line, allowing it to move freely but not escape. From a blind we recorded all behavior observed while the squirrel was within $3 \mathrm{~m}$ of the snake. The trial ended when the subject remained $>3 \mathrm{~m}$ from the snake for $>10 \mathrm{~min}$. We performed simple regression of offspring age against log-transformed behaviors displayed by mothers.

\section{Results}

As originally reported in an earlier paper (Swaisgood et al. 1999b), ground squirrel responses to rattling sounds were robust and sustained. After hearing rattle playbacks, ground squirrels adopted most of the responses typical of their interactions with live snakes. Most often, the squirrel backed away upon hearing the playback of rattling sounds, then stood bipedally and scanned the area before slowly reapproaching the speaker, pausing to tail flag as they reapproached.

Hypothesis 1: are maternal females more responsive to rattling playbacks than males and nonmaternal females?

Behavior among reproductive classes differed significantly (MANOVA: $F_{6,86}=2.2, P=0.047$ ). Planned comparisons showed that during rattling playback trials maternal females differed from nonmaternal females $(P=0.01)$, but not males, $(P=0.07)$. For purposes of contrast, we also compared males versus nonmaternal females, but as we predicted, this comparison was not significant $(P=0.28)$. Univariate ANOVAs, while not significant, suggest that tail-flagging accounts for much of these differences (males: $0.7 \pm 0.9$; mothers: $3.4 \pm 0.8$; nonmothers: $1 \pm 0.8 ; F_{2,16}=3.4, P=0.06$ ), whereas bipedal posture (males: 21.7 \pm 6.1 ; mothers: 22.4 \pm 6.4 ; nonmothers: $12.8 \pm 6.3 ; F_{2,16}=0.9, P=0.44$ ) and hesitancy to reapproach the playback speaker (males: 16.1 \pm 8.4 ; mothers: $24.5 \pm 7.2$; nonmothers: $\left.23.6 \pm 7.2 ; F_{2,16}=1.0, P=0.40\right)$ differed little among males, maternal females and nonmaternal females. Planned comparisons (including only data
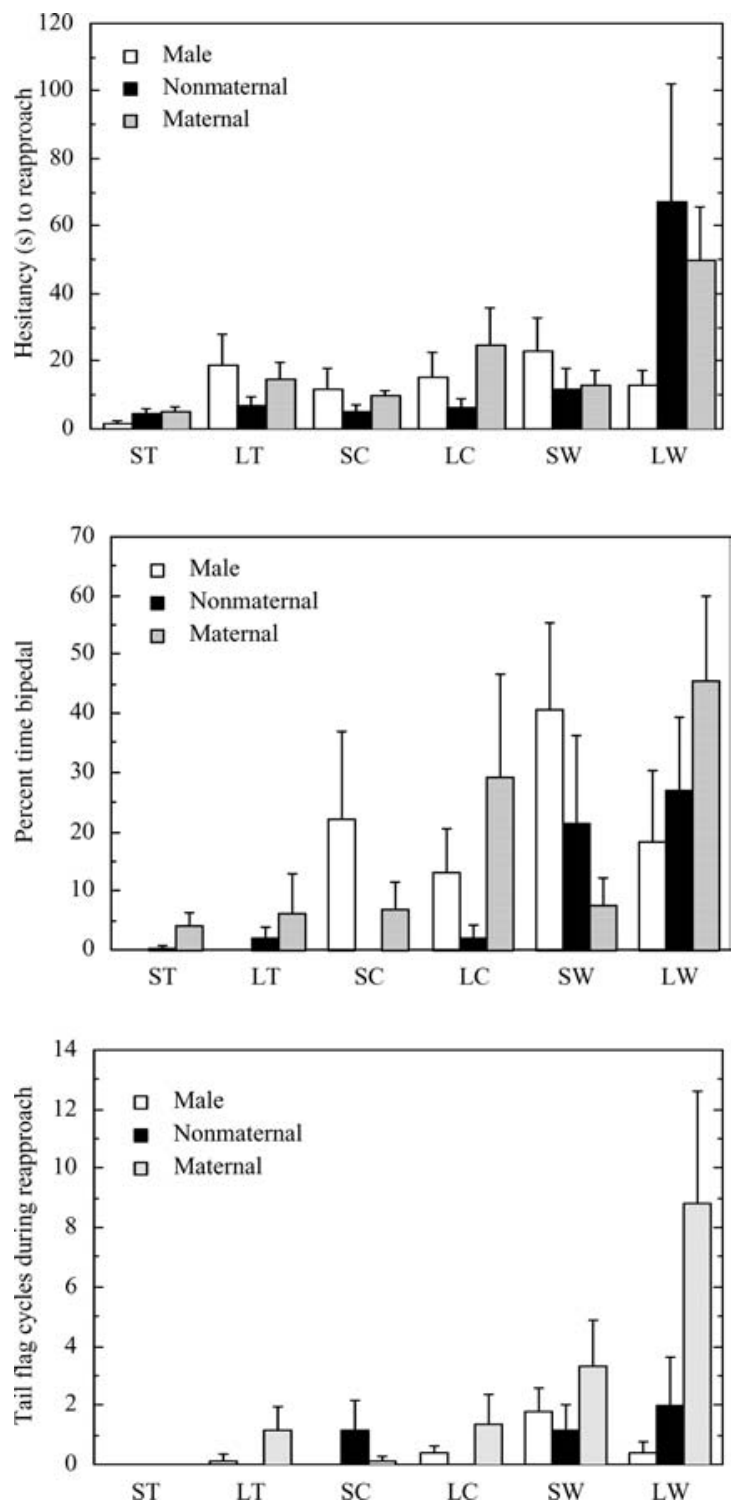

Fig. 1a-c Behavioral responses $(\overline{\mathrm{x}} \pm \mathrm{SE})$ to playbacks of rattling sounds to males, nonmaternal and maternal females. $S T=$ soft tone; $L T=$ loud tone; $S C=$ small cold snake rattle; $L C$ =large cold snake rattle; $S W=$ small warm snake rattle; $L W=$ large warm snake rattle. a Hesitancy (s) to reapproach the speaker following playback. b Percent time spent in bipedal posture. c Number of tail-flagging cycles during the reapproach to the playback speaker

from rattling trials) revealed that these reproductive classes did not differ significantly with regard to hesitancy to reapproach the speaker or bipedal posture, but maternal females tail flagged significantly more than males $(P=0.003)$ and nonmaternal females $(P=0.005)$. 
Table 1 Summary of results from planned comparisons for playback sounds examined separately for each reproductive class. Asterisk indicates significant tests $(P<0.05)$. In all cases, significant values are in the direction predicted, that is, heightened responses to rattles vs. tones, warm vs. cold snake rattling sounds, and large vs. small snake rattling sounds

\begin{tabular}{llll}
\hline Dependent variable & Tone versus rattle & Warm versus cold & Large versus small \\
\hline & Male & & \\
Hesitancy to reapproach & $P=0.22$ & $P=0.74$ & $P=0.83$ \\
Bipedal posture & $P=0.004^{*}$ & $P=0.23$ & $P=0.27$ \\
Tail-flag cycles & $P=0.28$ & $P=0.31$ & $P=0.68$ \\
& Maternal female & & \\
Hesitancy to reapproach & $P=0.0497^{*}$ & $P=0.056$ & $P=0.04^{*}$ \\
Bipedal posture & $P=0.03^{*}$ & $P=0.60$ & $P=0.002^{*}$ \\
Tail-flag cycles & $P=0.002^{*}$ & $P<0.0001^{*}$ & $P=0.04^{*}$ \\
& Nonmaternal female & & \\
Hesitancy to reapproach & $P=0.049^{*}$ & $P=0.002^{*}$ & $P=0.01^{*}$ \\
Bipedal posture & $P=0.14$ & $P=0.01^{*}$ & $P=0.48$ \\
Tail-flag cycles & $P=0.09$ & $P=0.21$ & $P=0.60$ \\
\hline
\end{tabular}

Fig. 2a-d The effects of offspring age on maternal risktaking in response to rattlesnakes and rattling sounds. a Average distance to the speaker following playback of rattling sounds from large warm rattlesnakes. b Time spent reapproaching the playback speaker after playback of rattling sounds from large warm rattlesnakes. c Time spent in proximity with $(\leq 3 \mathrm{~m})$ live tethered rattlesnakes. d Average distance to live tethered rattlesnakes while within $3 \mathrm{~m}$ of the snake
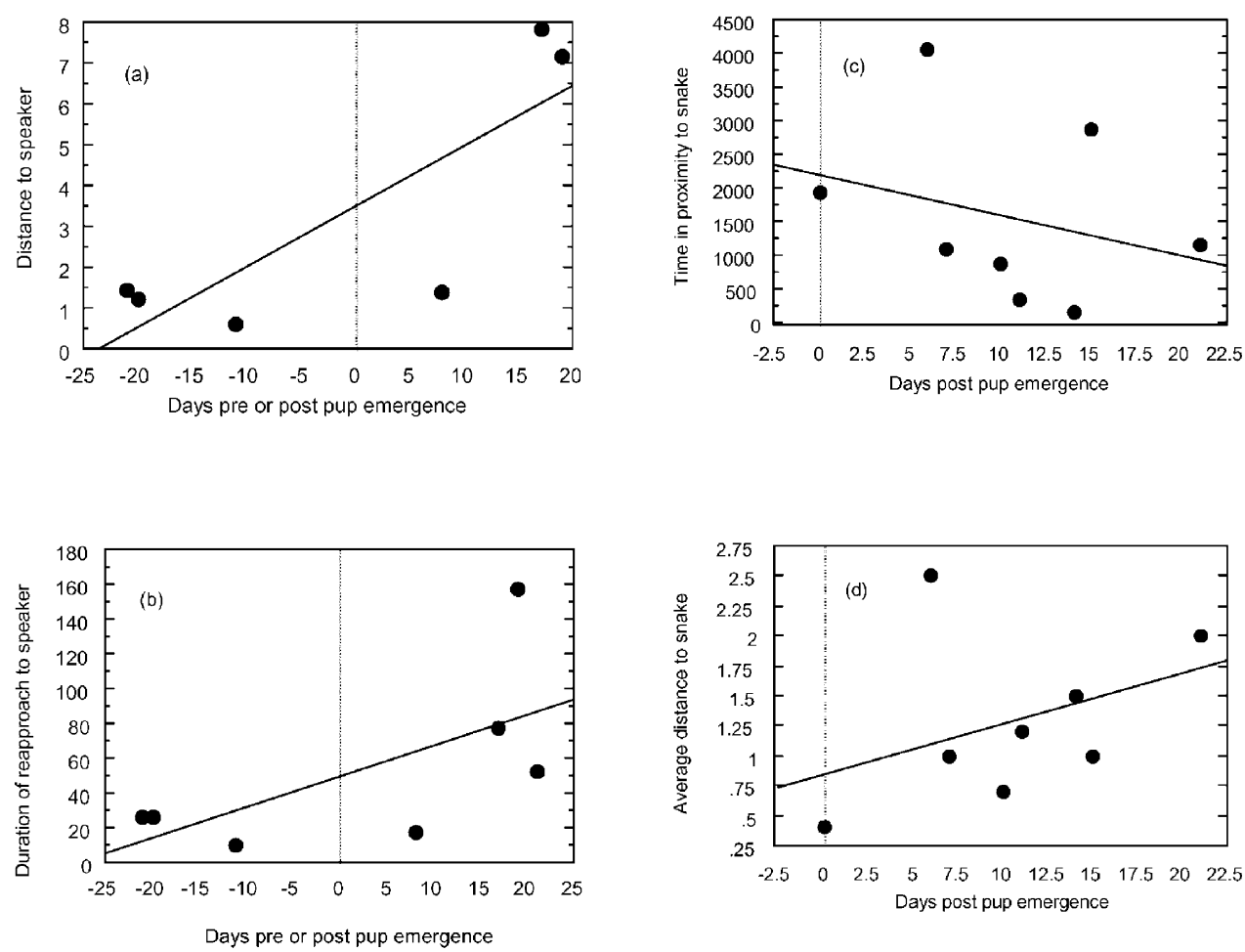

Hypothesis 2: are maternal females more sensitive to risk-related variation in playback sounds than males and nonmaternal females?

Behavioral discrimination among playback sounds differed significantly as a function of reproductive class (MANOVA reproductive class $\times$ playback interaction: $F_{30,135}=1.6, P=0.04$; Fig. 1). Mothers significantly discriminated among playback sounds for all three planned comparisons (tone vs. rattle: $P=0.004$; large vs. small: $P=0.0004$; warm vs. cold: $P=0.0001)$. By contrast males only overtly discriminated tones from rattles $(P=0.009)$ and nonmothers only differentiated cold from warm rattle playbacks $P=0.01$ ).

Of the three behavioral measures, only tail-flagging activity yielded a significant reproductive class $\times$ playback interaction (ANOVA: $F_{10,48}=2.6, P=0.01$ ). Planned comparisons show that mothers strongly differentiate playback sounds with their tail-flagging activity (Table 1). Mothers tail flagged more to rattles than tones, warm snake rattles than cold snake rattles, and large snake rattles than small snake rattles. By contrast, males and nonmothers did not discriminate among playback sounds on the basis of their tail-flagging activity. These differences in discrimination patterns among reproductive classes were less pronounced for the behavioral variables "hesitancy to reapproach the playback speaker" and "bipedal posture." Mothers were more hesitant to reapproach the speaker in response to (1) rattles than tones, (2) warm snake rattles than cold snake rattles (albeit not significant: $P=0.056$ ), and (3) large snake rattles than small snake rattles. Nonmother planned comparisons showed the same pattern, but all three were significant, whereas males took the same amount of time to 
reapproach the playback speaker regardless of the nature of the playback sound. Mothers spent more time standing bipedally in response to rattles than tones and large snake rattles than cold snake rattles. Nonmothers stood bipedally more following playback of warm snake rattles than cold snake rattles, while males stood bipedally more after rattles than tones. In sum, of the nine planned comparisons, mothers attained statistical significance for seven, nonmothers for four, and males for only one.

Hypothesis 3: does antisnake risk-taking among maternal females increase or decrease as offspring age?

If maternal responses to rattlesnakes and rattling sounds are governed by offspring value (increasing with increasing offspring age), then mothers should invest more in defense as their pups grow older. However, in response to playbacks of rattling sounds from large warm rattlesnakes maternal female ground squirrels remained significantly farther away from the playback speaker with increasing offspring age (simple regression: $n=7 ; r^{2}=0.67 ; P=0.047$; Fig. 2a), suggesting that mothers are less inclined to engage in risky behavior when they have older pups. With older offspring they also progressed somewhat more slowly while reapproaching the playback speaker, an effect that was not significant $\left(n=7 ; r^{2}=0.40 ; P=0.13\right.$; Fig. 2b). The relationships between pup age and their mothers' antisnake behavior in response to live snakes, while not significant, were also inconsistent with the offspring value hypothesis. Time spent in proximity $(3 \mathrm{~m})$ to the snake and average distance to the snake while in proximity were not associated with pup age $(n=8$; $r^{2}=0.08 ; P=0.49$; Fig. $2 \mathrm{c}$; and $r^{2}=0.15 ; P=0.34$; Fig. $2 \mathrm{~d}$, respectively). Indeed weak trends evident in Fig. $2 \mathrm{c}$ and $\mathrm{d}$ suggest the opposite: if anything, as offspring grow older mothers remain farther away from the tethered snake and spend less time in proximity with the snake, where presumably they could better monitor or dissuade the snake. These data suggest that with larger sample sizes results would show either no association between maternal risk-taking and offspring age or perhaps decreased risk-taking.

\section{Discussion}

How does sex and parity influence responses to rattling sounds?

Reproductive classes differed significantly in the MANOVA model, with mothers differing significantly from nonmothers, providing support for the hypothesis that mothers should be more responsive to acoustic cues from rattlesnakes. This effect was driven largely by maternal female tail flagging. Mothers tail flagged significantly more than males and nonmothers. Tail flagging is a conspicuous activity well designed for signal function. Maternal tail flagging does not necessarily indicate that this signal is directed toward pups. It is common before pup emergence (Hersek and Owings 1993), and was common in the playback trials, when pups were rarely above ground. Tail flagging might recruit adult squirrels to mob the snake or become more vigilant "spotters" (Hersek and Owings 1993). The snake might also be a target of tail flagging, which may amplify the effects of other snake-directed activities such as close approach and substrate throwing, and serve a pursuit deterrent function (sensu Hasson 1991). These activities may encourage the snake to leave by advertising the costs associated with the prey's (or its mother's) efforts to monitor and perhaps harass the snake (Swaisgood et al. 1999a). Rattlesnakes are highly cryptic sit-and-wait ambush predators, and allowing one to remain in the area-or losing track of its location-could and does prove fatal to pups (personal observations). In contrast, adults risk injury, not death, if they stumble onto a rattlesnake, which-because adults are not prey-would only strike an adult if threatened.

Consistent with the general lack of paternal care in California ground squirrels, male responses to rattling playbacks seem related to self preservation: they stood bipedally and engaged in cautious assessment, but signaled less in comparison with mothers. In a previous study, we were unable to find any evidence of paternal defense of young (Swaisgood et al. 1999a). Such lack of paternal care may reflect the high level of paternity uncertainty in the species (Boellstorff et al. 1994) or, alternatively, because mammalian mothers have greater opportunity for parental care because of their association with offspring at birth (Trivers 1972). The former hypothesis is supported by comparison with black-tailed prairie dogs, where harem-defending males have greater paternity certainty and invest more time and energy in antisnake behavior than do mothers (Loughry 1987; Loughry 1993).

Reproductive classes also differed markedly in their overt behavioral differentiation between the playback sounds, as suggested by a significant playback $\times$ reproductive class interaction in the MANOVA. Planned comparisons for each of the three behaviors, when significant, were always in the direction predicted, that is, heightened responses to acoustic cues from more dangerous snakes. Males displayed little evidence of discrimination, only differentiating tones from rattles on the basis of bipedal posture. Nonmothers were more discriminating: they were slower to reapproach the playback speaker following playback of rattles versus tones, warm versus cold snake rattles, and large versus small snake rattles, and stood bipedally longer in response to warm versus cold snake rattles. Mothers were the most discriminating. Of the nine planned comparisons for mothers, seven were significant, and they were the only reproductive class to differentiate between playback sounds with their tail-flagging activity. Mothers' tail flagging was most dramatically elevated following rattling sounds from large warm snakes, the most dangerous and most likely snake to be hunting pups in the colony. Why is it so important for mothers to track snake 